\title{
Angiopoietin-like protein 2 in psoriasis: a new linkage with metabolic syndrome
}

\author{
Mohamed Zaki Kenawy¹, Jehan Hasan Sabry², Essam Mohamed Akl ${ }^{1}$, Noha A.H. Emam ${ }^{1}$
}

${ }^{1}$ Department of Dermatology, Venereology and Andrology, Faculty of Medicine, Benha University, Benha, Egypt ${ }^{2}$ Department of Clinical Pathology, Faculty of Medicine, Benha University, Benha, Egypt

Adv Dermatol Allergol 2020; XXXVII (1): 86-91

DOI: https://doi.org/10.5114/ada.2018.79225

\begin{abstract}
Introduction: Psoriasis is a chronic disease of inflammatory nature which can be considered as a systemic disorder. Metabolic syndrome is prevalent in psoriatic patients, with a negative impact on disease severity. Angiopoietin-like protein 2 (ANGPTL2) role has been investigated in several chronic inflammatory conditions, but not in psoriasis. Aim: To evaluate the serum level of ANGPTL2 and its possible role in the occurrence of metabolic syndrome in psoriatic patients.

Material and methods: This study enrolled 180 participants divided into two groups: psoriatic group (120 patients with chronic plaque psoriasis) and control group (60 normal subjects). Psoriasis severity was determined by the psoriasis area severity index. Anthropometric measurements, lipid profile, fasting blood sugar and ANGPTL2 have been evaluated in both groups.

Results: Psoriatic patients had a higher body mass index $(p=0.014)$, waist circumference $(p<0.001)$, and blood pressure than controls $(p \leq 0.001)$. Fasting blood sugar and the serum level of ANGPTL2 were also higher in psoriatic patients than in controls ( $p<0.001,0.025$, respectively). In addition, the serum level of ANGPTL2 was significantly correlated with both disease severity $(p<0.001)$ and occurrence of metabolic syndrome $(p<0.001)$.

Conclusions: Serum ANGPTL2 is elevated in psoriasis patients compared to normal subjects. Serum ANGPTL2 elevation may have a role in chronic inflammatory status in psoriasis and occurrence of metabolic syndrome.
\end{abstract}

Key words: psoriasis, obesity, metabolic syndrome, angiopoietin-like protein 2.

\section{Introduction}

Psoriasis is a common chronic inflammatory dermatological disease [1]. Due to the association with several co-morbidities such as cardiovascular disease, atherosclerosis, diabetes mellitus, obesity, psychiatric disorders and metabolic syndrome [2]; psoriasis can be now recognised as a systemic and not only a skin disorder [1]. Obesity is more commonly seen in psoriatic patients than in the general population [3], but whether obesity antedates or occurs after the development of psoriasis is unclear [4]. Obesity is known as a risk factor for incident psoriasis and can even aggravate the existing disease. In addition, weight reduction improved psoriasis in some overweight patients [5].

Angiopoietin-like protein 2 (ANGPTL2) is a glycoprotein, mainly secreted from adipose tissue [6]. Acting as a mediator of chronic inflammation; ANGPTL2 has contributed to the pathogenesis of several chronic diseas- es [7-10]; however, there are no previous studies about ANGPTL2 role in psoriasis.

\section{Aim}

The aim of the present work was to evaluate the serum level of ANGPTL2 and its possible role in the occurrence of metabolic syndrome in psoriatic patients.

\section{Material and methods}

\section{Ethical approval}

The Research Ethics Committee, Faculty of Medicine, Benha University, Egypt, had approved this study before the proceeding of the work. Written informed consent was obtained from all participants and they were not exposed to any harmful effects. The patient's personal data were secured and not published.

Address for correspondence: Essam Mohamed Akl, Department of Dermatology, Venereology and Andrology, Faculty of Medicine, Benha University, Benha, Egypt, e-mail: esamakl@hotmail.com Received: 3.07.2018, accepted: 8.08.2018. 


\section{Type of the study}

A cross-sectional case-control comparative study.

\section{Material}

This study included 120 patients with chronic plaque psoriasis (confirmed by grattage test clinically and histopathologically if needed), and 60 healthy, age- and sexmatched controls. This study was conducted between November 2017 and April 2018.

Participants included in this study were older than 18 years of age, non-pregnant, free of chronic haematological, renal, hepatic, infectious or neoplastic diseases. Patients suffering from chronic plaque psoriasis only were included in this study; other types of psoriasis were excluded. The Psoriasis Area Severity Index (PASI) scoring formula was applied to estimate the severity of psoriasis [11]. Psoriatic patients with more than 6 months' duration and PASI score of more than eight were considered eligible for the study. The participating psoriatic patients were instructed to stop using any systemic, phototherapy or topical treatment for one month before the study.

\section{Methods}

On enrolment, body mass index (BMI), waist circumference (WC) and blood pressure of all participants were recorded. BMI was estimated in weight/height ${ }^{2}\left(\mathrm{~kg} / \mathrm{m}^{2}\right)$. Participants were classified according to $\mathrm{WHO}$ guidelines into underweight (BMI $<18.5 \mathrm{~kg} / \mathrm{m}^{2}$ ), normal-weight (BMI $\left.=18.5-24.9 \mathrm{~kg} / \mathrm{m}^{2}\right)$, overweight $\left(\mathrm{BMI}=25-29.9 \mathrm{~kg} / \mathrm{m}^{2}\right)$ or obese $\left(\mathrm{BMI} \geq 30 \mathrm{~kg} / \mathrm{m}^{2}\right)$ [12]. A mid-point between the lower borders of the rib cage and the iliac crest of the participants was used to measure WC.

To diagnose the presence of metabolic syndrome, the National Cholesterol Education Program's Adult Treatment Panel III (NCEP ATP III) revised clinical criteria were applied [13]. These criteria included five laboratory and clinical features and the presence of three of them was sufficient for a diagnosis of metabolic syndrome. The laboratory criteria included an elevation of fasting blood sugar (FBS) and serum triglycerides (more than $100 \mathrm{mg} / \mathrm{dl}, 150 \mathrm{mg} / \mathrm{dl}$, respectively), and a level of serum high-density lipoprotein cholesterol (HDL-cholesterol) of less than $40 \mathrm{mg} / \mathrm{dl}$ in men and $50 \mathrm{mg} / \mathrm{dl}$ in women or receiving drug therapy for these changes. Clinical criteria were an elevation of blood pressure of more than 130/ $85 \mathrm{~mm} \mathrm{Hg}$ or receiving antihypertensive therapy and a WC of more than $102 \mathrm{~cm}$ (40 inches) in men or $88 \mathrm{~cm}$ (35 inches) in women [13].

\section{Laboratory investigations}

Venous blood samples were withdrawn from the participants in the morning after an overnight fasting. After clot formation, samples were centrifuged at 2,000 $\mathrm{g}$ for 10 min. Routine biochemical parameters were measured on the same day. Remaining serum samples were stored at $-20^{\circ} \mathrm{C}$ until the day on which they were analysed for the concentration of ANGPTL2.

The Frigewal's formula was used to calculate lowdensity lipoprotein cholesterol (LDL-cholesterol) [14], while the serum ANGPTL2 level was determined using the commercially available ELISA human monoclonal antibody kits (Clone 239829, MAB2084, R\&D systems Inc., Minneapolis, MN, USA) according to the manufacturer's manuals.

\section{Statistical analysis}

The independent $T$-test was used to compare the numerical data of normally distributed variables, while categorical variables differences were analysed with $\chi^{2}$ (chisquare) test. Spearman correlation was used to examine correlations. If the $p$-values were $\leq 0.05$, it was considered significant. The statistical analysis was achieved using the SPPS ${ }^{\oplus}$ program for Microsoft Windows $7^{\oplus}$.

\section{Results}

This study included 180 subjects, 120 psoriatic patients (65 males and 55 females) with age ranging from 19 to 60 years and 60 normal subjects ( 32 males and 28 females) with age ranging from 21 to 58 years as a control group. Diabetes mellitus (DM) was diagnosed in 39 psoriatic patients and 8 in the control group of all the participants, and these diabetic patients were under oral hypoglycaemic therapy. Their demographic, clinical and laboratory data are presented in Table 1.

By comparison, there was no significant difference regarding age and sex between the two groups $(p>0.05$, Table 1). Regarding physical data, mean BMI, WC, systolic and diastolic blood pressure were higher in the psoriatic patients than controls ( $p \leq 0.05$, Table 1$)$. Regarding laboratory data, both FBS and ANGPTL2 were significantly higher in patients than controls ( $p \leq 0.05$, Table 1$)$. On the other hand, there were no significant differences between patients and controls regarding serum levels of HDL, LDL, triglycerides, and cholesterol ( $p>0.05$, Table 1).

Among the psoriatic group, 45 patients fulfilled the NCEP ATP III clinical criteria to be diagnosed as having metabolic syndrome, while 12 subjects in the control group fulfilled these criteria, with a significant difference between both groups ( $p \leq 0.05$, Table 1$)$.

The serum level of ANGPTL2 was significantly higher in psoriatic patients than controls in different BMI groups ( $p \leq 0.05$, Table 2 ). In addition, psoriatic patients suffering from DM had a higher serum level of ANGPTL2 than diabetic control participants ( $p \leq 0.05$, Table 2 ).

Using correlation analysis in the studied participants, there were positive significant correlations between the mean serum ANGPTL2 level and BMI, WC, serum cholesterol and LDL $(p \leq 0.05)$, but not with systolic and diastolic blood pressure, FBS, serum triglycerides or $\mathrm{HDL}$ ( $p>0.05$, Table 2). 
Table 1. Demographic, clinical and laboratory data of psoriatic patients and controls involved in this study

\begin{tabular}{|c|c|c|c|}
\hline Variables & $\begin{array}{l}\text { Patients } \\
(n=120)\end{array}$ & $\begin{array}{l}\text { Controls } \\
(n=60)\end{array}$ & $P$-value \\
\hline Age [years] & $42.7 \pm 12.1$ & $37.8 \pm 9.7$ & 0.06 \\
\hline \multicolumn{3}{|l|}{ Sex: } & \multirow[t]{3}{*}{0.916} \\
\hline Male & 65 & 32 & \\
\hline Female & 55 & 28 & \\
\hline $\mathrm{BMI}\left[\mathrm{kg} / \mathrm{m}^{2}\right]$ & $28.0 \pm 5.1$ & $25.2 \pm 4.5$ & $0.014^{*}$ \\
\hline WC [inch] & $39.6 \pm 4.6$ & $35.8 \pm 4.1$ & $<0.001^{*}$ \\
\hline Systolic BP [mm Hg] & $128.6 \pm 17.4$ & $111 \pm 16.9$ & $<0.001^{*}$ \\
\hline Diastolic BP [mm Hg] & $81.6 \pm 8.9$ & $73.7 \pm 10$ & $<0.001^{*}$ \\
\hline FBS [mg/dl] & $127.4 \pm 59.3$ & $96.3 \pm 8.8$ & $0.001^{*}$ \\
\hline S. Ch [mg/dl] & $178.3 \pm 28.5$ & $176.2 \pm 28.8$ & 0.751 \\
\hline S. TG [mg/dl] & $75 \pm 22.4$ & $74.6 \pm 15$ & 0.918 \\
\hline $\mathrm{HDL}[\mathrm{mg} / \mathrm{dl}]$ & $44.6 \pm 7.9$ & $44.7 \pm 7.9$ & 0.956 \\
\hline LDL [mg/dl] & $117.7 \pm 25.4$ & $114.4 \pm 24.2$ & 0.566 \\
\hline Metabolic syndrome (yes/no) & $45 / 75$ & $12 / 48$ & $0.0173^{*}$ \\
\hline Serum ANGPTL2 [ng/ml] & $9.54 \pm 10.47$ & $4.94 \pm 5.14$ & $0.025^{\star}$ \\
\hline Duration of disease [years] & $7.4 \pm 4.8$ & & \\
\hline PASI score & $17.27 \pm 7.9$ & & \\
\hline
\end{tabular}

Value data are presented as mean $\pm S D .{ }^{*} P \leq 0.05$ is significant, $p>0.05$ is non-significant. Metabolic syndrome: fulfilled/not fulfilled National Cholesterol Education Program's Adult Treatment Panel III (NCEP ATP III) revised clinical criteria for metabolic syndrome. ANGPTL2 - angiopoietin-like protein 2, BMI - body mass index, BP-blood pressure, FBS - fasting blood sugar, $H D L$ - high-density lipoprotein, $L D L$ - low-density lipoprotein, PASI - psoriasis area and severity index, S. Ch-serum cholesterol, S. TG - serum triglyceride, WC - waist circumference.

Table 2. Comparison of serum ANGPTL2 [ng/ml] levels between psoriatic patients and controls regarding different BMI and DM

\begin{tabular}{lccc}
\hline Paramater & Psoriatic patients & Control & $P$-value \\
\hline BMI: & & & \\
\hline Underweight $\left(\mathrm{BMI}<18.5 \mathrm{~kg} / \mathrm{m}^{2}\right)$ & $6.07 \pm 4.43(n=18)$ & $3.32 \pm 2.56(n=14)$ & $0.025^{*}$ \\
\hline Normal weight $\left(\mathrm{BMI}=18.5-24.9 \mathrm{~kg} / \mathrm{m}^{2}\right)$ & $7.99 \pm 11.95(n=24)$ & $5.67 \pm 6.56(n=15)$ & $0.007^{\star}$ \\
\hline Overweight $\left(\mathrm{BMI}=25-29.9 \mathrm{~kg} / \mathrm{m}^{2}\right)$ & $9.45 \pm 11.94(n=41)$ & $5.67 \pm 6.56(n=21)$ & $0.023^{\star}$ \\
\hline Obese $\left(\mathrm{BMI} \geq 30 \mathrm{~kg} / \mathrm{m}^{2}\right)$ & $12.834 \pm 13.43(n=37)$ & $5.92 \pm 6.79(n=10)$ & $0.0178^{*}$ \\
\hline DM: & $12.85 \pm 8.43(n=39)$ & $5.63 \pm 4.45(n=8)$ & $0.042^{*}$ \\
\hline Diabetic & $11.75 \pm 7.48(n=81)$ & $4.63 \pm 3.85(n=52)$ & $<0.001^{*}$ \\
\hline Non-diabetic
\end{tabular}

Value data are presented as mean $\pm S D$. ${ }^{*} P \leq 0.05$ is significant, $p>0.05$ is non-significant. ANGPTL2-angiopoietin-like protein 2 , BMI-body mass index, DM - diabetes mellitus.

Analysing the psoriatic group only, there was a significant positive correlation found between the serum ANGPTL2 level and PASI score (Figure 1), BMI, WC, systolic and diastolic blood pressure, serum cholesterol, LDL and metabolic syndrome $(p>0.05)$, but not with FBS, serum triglycerides or HDL ( $p \leq 0.05$, Table 3$)$.

Analysing the psoriatic group as well, there was a significant positive correlation found between PASI score 


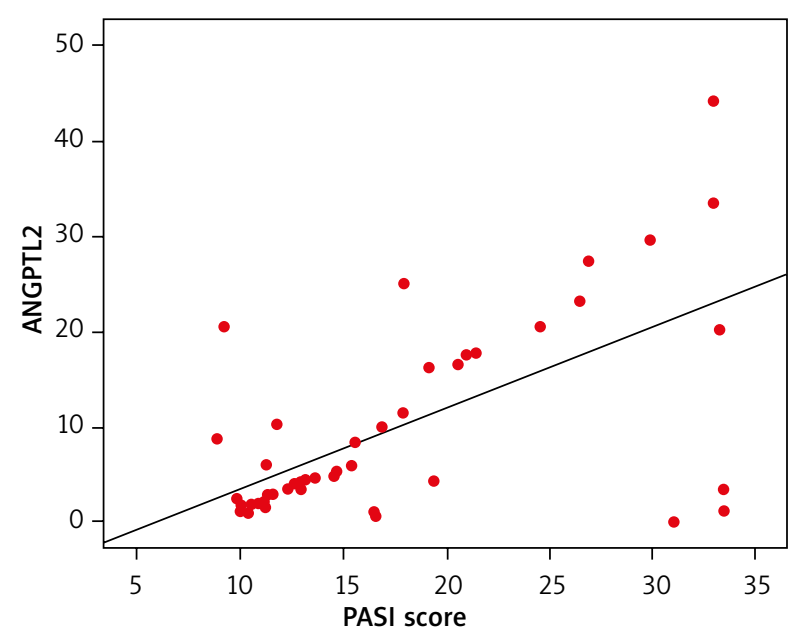

Figure 1. Correlation between the serum level of ANGPTL2 and PASI score

and BMI, WC, systolic and diastolic blood pressure, serum cholesterol, LDL and metabolic syndrome $(p \leq 0.05)$, but not with FBS, serum triglycerides or $\mathrm{HDL}(p>0.05$, Table 4).

\section{Discussion}

Angiopoietin-like protein-2 (ANGPTL2) is a secretory glycoprotein [15], primarily secreted from adipose tissues with a higher expression detected in obesity and obesityrelated pathological diseases [16]. The inflammatory role of ANGPTL2 in adipose tissue is through the recruitment of macrophages and inflammatory vascular remodelling [9]. In an experimental study, epidermis-specific ANGPTL2-transgenic mice had an adherent leukocyte increment in enlarged vessels and increased permeability of the skin [17], which is similar to the vascular changes seen in psoriasis pathologically [18]. In addition to adipocytes, several cells that are involved in psoriasis pathogenesis can secrete ANGPTL2, such as macrophages [19], keratinocytes [8] and endothelial cells [20].

The results of this study revealed that serum ANGPTL2 is elevated in psoriatic patients than controls, and significant correlations with both psoriasis severity (PASI) and presence of metabolic syndrome, which may indicate its potential role in psoriasis pathogenesis. The main source of circulating serum ANGPTL2 is adipose tissue, and an increase was observed in obesity, systemic insulin resistance and low-grade systemic inflammation [17], which is usually seen in psoriasis [3].

Psoriasis is a complex chronic inflammatory dermatological disease and can be considered as a systemic and not only skin disorder [21]. As shown in this study, psoriatic patients had a higher BMI than normal subjects; this could be explained by the role of adipokines, which appear in both psoriasis and obesity [22] and contribute to the inflammatory state seen in chronic psoriasis [23].
Table 3. Correlation between the serum level of ANGPTL2 and different variables in the participants of the study

\begin{tabular}{lcccc}
\hline Variable & \multicolumn{2}{c}{$\begin{array}{c}\text { Psoriatic patients } \\
\text { only }\end{array}$} & \multicolumn{2}{c}{ All participants } \\
\cline { 2 - 5 } & $r$ & $P$-value & $r$ & $P$-value \\
\hline Age & -0.193 & 0.18 & -0.014 & 0.901 \\
\hline BMI & 0.522 & $<0.001^{*}$ & 0.544 & $<0.001^{*}$ \\
\hline WC & 0.454 & $<0.001^{*}$ & 0.448 & $<0.001^{*}$ \\
\hline Systolic BP & 0.359 & $0.003^{\star}$ & 0.259 & 0.389 \\
\hline Diastolic BP & 0.376 & $0.004^{\star}$ & 0.139 & 0.217 \\
\hline FBS & -0.062 & 0.669 & 0.039 & 0.731 \\
\hline S. Ch & 0.346 & $0.014^{*}$ & 0.329 & $0.003^{*}$ \\
\hline S. TG & -0.113 & 0.433 & -0.118 & 0.298 \\
\hline HDL & -0.169 & 0.241 & -0.146 & 0.197 \\
\hline LDL & 0.483 & $<0.001^{*}$ & 0.417 & $<0.001^{*}$ \\
\hline Metabolic syndrome & 0.489 & $<0.001^{*}$ & 0.387 & $0.006^{*}$ \\
\hline Duration of psoriasis & -0.107 & 0.46 & & \\
\hline PASI score & 0.503 & $<0.001^{*}$ & & \\
\hline
\end{tabular}

$r$-Pearson correlation coefficient. * $(P \leq 0.05)$ is significant; $(p>0.05)$ is nonsignificant. BMI - body mass index, BP - blood pressure, FBS - fasting blood sugar, HDL - High-density lipoprotein, LDL - Low-density lipoprotein, S. Chserum cholesterol, S. TG - serum triglyceride, WC - waist circumference.

Table 4. Correlation between PASI and different variables in the psoriatic patients

\begin{tabular}{lcc}
\hline Variable & $r$ & $P$-value \\
\hline Age & -0.032 & 0.852 \\
\hline Duration of disease & 0.067 & 0.646 \\
\hline BMI & 0.493 & $<0.001^{*}$ \\
\hline WC & 0.683 & $<0.001^{*}$ \\
\hline Systolic BP & 0.459 & $0.002^{\star}$ \\
\hline Diastolic BP & 0.476 & $0.005^{\star}$ \\
\hline FBS & -0.076 & 0.599 \\
\hline S. Ch & 0.395 & $0.005^{\star}$ \\
\hline S. TG & -0.221 & 0.123 \\
\hline HDL & 0.017 & 0.907 \\
\hline LDL & 0.47 & $<0.001^{*}$ \\
\hline Metabolic syndrome & 0.389 & $<0.001^{*}$
\end{tabular}

$r$-Pearson correlation coefficient. ${ }^{*} P \leq 0.05$ is significant; $p>0.05$ is nonsignificant. BMI-body mass index, BP-blood pressure, FBS - fasting blood sugar, HDL - high-density lipoprotein, LDL - low-density lipoprotein, PASI psoriasis area and severity index, S. Ch - serum cholesterol, S. TG - serum triglyceride, WC - waist circumference.

Psoriatic patients in our study had a higher WC than controls, and this indicates an increase in abdominal fat. As a wide variety of soluble mediators termed 'adipokines' that play important roles in chronic inflammation, 
the white adipose tissue is considered as an active endocrine organ [24]. Amidst a chronic inflammatory milieu [25], psoriasis is characterized by elevation of several cytokines such as tumour necrosis factor $\alpha$ (TNF- $\alpha$ ) [26], which in turn regulates ANGPTL2 mRNA expression [27]. In addition, adding obesity may act on the elevation of the ANGPTL2 serum level observed in this study.

Angiopoietin-like protein-2 can, through several pathways such as activation of the nuclear factor $\kappa B(N F-\kappa B)$, induce endothelial cell inflammation, expression of proinflammatory cytokines such as interleukin-6 (IL-6) and TNF- $\alpha$ and adhesion molecule, which maintain a chronic inflammatory status [28], and these molecules also have roles in psoriasis pathogenesis [29].

Our results showed that metabolic syndrome is more prevalent in psoriatic patients than controls, and that is in concordance with other studies [30,31]. Factors involved in metabolic syndrome are connected with each other, and most of them have been found to be independently associated with psoriasis [2].

This study included generalized plaque psoriasis patients only; other types of psoriasis were excluded, and the serum level of ANGPTL2 was not measured before and after any psoriasis treatment.

\section{Conclusions}

The results of this study showed that the serum ANGPTL2 level is elevated in psoriatic patients compared to controls with positive correlations with psoriasis severity, BMI, WC, serum cholesterol LDL and presence of metabolic syndrome. It seems that ANGPTL2 has a role in the chronic inflammatory state of psoriasis and associated comorbidity. In addition, ANGPTL2 can be considered as a laboratory biomarker of both psoriasis severity and metabolic syndrome.

\section{Conflict of interest}

The authors declare no conflict of interest.

\section{References}

1. Takeshita J, Grewal S, Langan SM, et al. Psoriasis and comorbid diseases: epidemiology. J Am Acad Dermatol 2017; 76: 377-90.

2. Lønnberg AS, Skov L. Co-morbidity in psoriasis: mechanisms and implications for treatment. Expert Rev Clin Immunol 2017; 13: 27-34.

3. Coimbra S, Catarino C, Santos-Silva A. The triad psoriasisobesity-adipokine profile. J Eur Acad Dermatol Venereol 2016; 30: 1876-85.

4. Ryan C, Kirby B. Psoriasis is a systemic disease with multiple cardiovascular and metabolic comorbidities. Dermatol Clin 2015; 33: 41-55.

5. Jensen P, Skov L. Psoriasis and obesity. Dermatology 2016; 232: 633-9.
6. Carbone C, Piro G, Merz V, et al. Angiopoietin-like proteins in angiogenesis, inflammation and cancer. Int J Mol Sci 2018; 19: pii: E431

7. Ide S, Toiyama Y, Shimura T, et al. Angiopoietin-like protein 2 acts as a novel biomarker for diagnosis and prognosis in patients with esophageal cancer. Ann Surg Oncol 2015; 22: 2585-92.

8. Ogata A, Endo M, Aoi J, et al. The role of angiopoietin-like protein 2 in pathogenesis of dermatomyositis. Biochem Biophys Res Commun 2012; 418: 494-9.

9. Okada T, Tsukano H, Endo M, et al. Synoviocyte-derived angiopoietin-like protein 2 contributes to synovial chronic inflammation in rheumatoid arthritis. Am J Pathol 2010; 176: 2309-19.

10. Morinaga J, Kadomatsu T, Miyata K, et al. Angiopoietin-like protein 2 increases renal fibrosis by accelerating transforming growth factor-beta signaling in chronic kidney disease. Kidney Int 2016; 89: 327-41.

11. Henseler T, Schmitt-Rau K. A comparison between BSA, PASI, PLASI and SAPASI as measures of disease severity and improvement by therapy in patients with psoriasis. Int J Dermatol 2008; 47: 1019-23.

12. World Health Organization. Physical Status: the Use and Interpretation of Anthropometry. Geneva: World Health Organization; 1995: 1-452. WHO Technical Report Series no. 854.

13. Third Report of the National Cholesterol Education Program (NCEP) Expert Panel on detection, evaluation, and treatment of high blood cholesterol in adults (Adult Treatment Panel III) final report. Circulation 2002; 106: 3143-421.

14. Okan G, Baki AM, Yorulmaz E, et al. Serum visfatin, fetuin-a, and pentraxin 3 levels in patients with psoriasis and their relation to disease severity. J Clin Lab Anal 2016; 30: 284-9.

15. Kim I, Moon SO, Koh KN, et al. Molecular cloning, expression, and characterization of angiopoietin-related protein. angiopoietin-related protein induces endothelial cell sprouting. J Biol Chem 1999; 274: 26523-8.

16. Lee HJ, Kim JH, Kim JH, et al. Angiopoietin-like protein 2, a chronic inflammatory mediator, is a new target induced by TGF-beta1 through a Smad3-dependent mechanism. Biochem Biophys Res Commun 2013; 430: 981-6.

17. Tabata M, Kadomatsu T, Fukuhara S, et al. Angiopoietin-like protein 2 promotes chronic adipose tissue inflammation and obesity-related systemic insulin resistance. Cell Metab 2009; 10: $178-88$.

18. Perera GK, Di Meglio P, Nestle FO. Psoriasis. Annu Rev Pathol 2012; 7: 385-422.

19. Tazume H, Miyata K, Tian Z, et al. Macrophage-derived angiopoietin-like protein 2 accelerates development of abdominal aortic aneurysm. Arterioscler Thromb Vasc Biol 2012; 32: 1400-9.

20. Farhat N, Thorin-Trescases N, Mamarbachi M, et al. Angiopoietin-like 2 promotes atherogenesis in mice. J Am Heart Assoc 2013; 2: e000201.

21. Gerdes S, Rostami-Yazdi M, Mrowietz U. Adipokines and psoriasis. Exp Dermatol 2011; 20: 81-7.

22. Lynch M, Ahern T, Sweeney CM, et al. Adipokines, psoriasis, systemic inflammation, and endothelial dysfunction. Int J Dermatol 2017; 56: 1103-18.

23. Takahashi H, Tsuji H, Honma M, et al. Increased plasma resistin and decreased omentin levels in Japanese patients with psoriasis. Arch Dermatol Res 2013; 305: 113-6.

24. Booth A, Magnuson A, Fouts J, et al. Adipose tissue: an endocrine organ playing a role in metabolic regulation. Horm Mol Biol Clin Investig 2016; 26: 25-42. 
25. Boehncke WH, Boehncke S, Tobin AM, et al. The 'psoriatic march': a concept of how severe psoriasis may drive cardiovascular comorbidity. Exp Dermatol 2011; 20: 303-7.

26. Takahashi H, Tsuji H, Hashimoto Y, et al. Serum cytokines and growth factor levels in Japanese patients with psoriasis. Clin Exp Dermatol 2010; 35: 645-9.

27. Zheng JY, Zou JJ, Wang WZ, et al. Tumor necrosis factor-alpha increases angiopoietin-like protein 2 gene expression by activating Foxo1 in 3T3-L1 adipocytes. Mol Cell Endocrino 2011; 339: 120-9.

28. Umikawa M, Umikawa A, Asato T, et al. Angiopoietin-like protein 2 induces proinflammatory responses in peritoneal cells. Biochem Biophys Res Commun 2015; 467: 235-41.

29. Harden JL, Krueger JG, Bowcock AM. The immunogenetics of psoriasis: a comprehensive review. J Autoimmun 2015; 64: 66-73.

30. Armstrong AW, Harskamp CT, Armstrong EJ. Psoriasis and metabolic syndrome: a systematic review and meta-analysis of observational studies. J Am Acad Dermatol 2013; 68: 65462.

31. Singh S, Young P, Armstrong AW. Relationship between psoriasis and metabolic syndrome: a systematic review. G Ital Dermatol Venereol 2016; 151: 663-77. 